\title{
Systemic Ropivacaine Diminishes Pain Sensitization Processes: A Randomized, Double-Blinded, Placebo-Controlled, Crossover Study in Healthy Volunteers
}

Yéri Haller • Andreas R. Gantenbein • Patrick Willimann •

Donat R. Spahn · Konrad Maurer

To view enhanced content go to www.paintherapy-open.com Received: July 26, 2013 / Published online: January 3, 2014

(c) The Author(s) 2013. This article is published with open access at Springerlink.com

\section{ABSTRACT}

Introduction: Ropivacaine is a local anesthetic widely used for regional anesthesia. One of its advantages is low toxicity at plasma concentrations reached systemically during continuous peripheral or central nervous block. The objective of this study was to test the effect of

Electronic supplementary material The online version of this article (doi:10.1007/s40122-013-0021-z) contains supplementary material, which is available to authorized users.

Clinicaltrials.gov: \#NCT00900913.

Y. Haller · D. R. Spahn · K. Maurer $(\bowtie)$ Institute of Anaesthesiology, University Hospital of Zurich, Raemistrasse 100, 8091 Zurich, Switzerland e-mail: konrad.maurer@usz.ch

\section{A. R. Gantenbein}

Neurorehabilitation, RehaClinic Bad,

Quellenstrasse 34, Zurzach, 5330 Baden, Switzerland

\section{P. Willimann}

Hirslanden Klinik Aarau, Schänisweg,

5001 Aarau, Switzerland

\section{K. Maurer}

Institute of Physiology and Zurich Centre for Integrative Human Physiology (ZIHP),

University of Zurich, Zurich, Switzerland

K. Maurer

Clinical Trials Centre, University Hospital Zurich and University of Zurich, Zurich, Switzerland systemic ropivacaine on pain, hyperalgesia, dynamic allodynia, and flare response.

Methods: This randomized, double-blinded, placebo-controlled, crossover study was carried out in at the Clinical Trials Centre, University of Zurich, Switzerland. Twenty healthy male volunteers were included in the study. Exclusion criteria were contraindications or hypersensitivity to local anesthetics, vulnerable subjects (intellectually or mental impaired), drug, alcohol or nicotine abuse, known peripheral neuropathies, diabetes mellitus and/or congestive heart disease. Ropivacaine and saline were infused intravenously during a subcutaneous electrical stimulation. The stimulation software adjusted the stimulus strength according to the rating on a numeric rating scale (NRS; 0-10) maintaining a NRS of 5. Areas of punctate hyperalgesia, dynamic allodynia, and flare response were measured before and after the infusion.

Results: The area of hyperalgesia increased significantly with saline $(303 \pm 380 \%, P<0.05)$ and ropivacaine $(186 \pm 137 \%, P<0.05)$. The area of allodynia $(253 \pm 299 \%, P<0.05)$ and flare response $(112 \pm 24 \%, P<0.05)$ increased only during the placebo infusion. 
Conclusion: The results of this study imply that systemic ropivacaine may diminish pain sensitization processes.

Keywords: Allodynia; Electric pain model; Flare response; Hyperalgesia; Pain; Pain sensitization; Ropivacaine

\section{INTRODUCTION}

Local anesthetics play an important role in perioperative analgesia. By means of continuous peripheral or central nerve blocks, they can easily be effective for days with the appropriate management and a minimal risk of toxic effects $[1,2]$. Although the plasma concentrations measured after several days of continuous infusion increase considerably, they remain low enough to be tolerated [3].

A whole body of evidence suggests that not only do local anesthetics prevent the transmission of action potentials at high concentrations (local effect), but also they can modulate pain pathways at low plasma concentrations (systemic effect) $[4,5]$. This modulation involves direct interaction of the local anesthetic with neuronal tissue [6-8] as well as indirect mechanisms via a reduction of circulating pro-inflammatory cytokines $[9,10]$.

The pure $S$-enantiomer ropivacaine is widely used in clinical practice for its safety profile $[11,12]$ and the lowest potential for neurotoxicity [13]. Recently published data on the effect of low-dose ropivacaine on mechanisms of peripheral neurogenic inflammation concluded that ropivacaine may be useful in the suppression of inflammation, mechanical and visceral hypersensitivity [14, 15]. Some authors even consider ropivacaine to be a promising alternative to systemic lidocaine which is used to treat chronic pain states [16].
The aim of our study was to quantify the effect of low-dose ropivacaine on hyperalgesia, allodynia, and flare reaction in a model of electrically induced pain in healthy volunteers. This model allows the study of acute and secondary pain phenomenon evoked by electrical current administered on peripheral nerve endings in the skin. It has been used in earlier investigations to show that local anesthetics can prevent the development of these symptoms [7, 17]. The information gained from this study might be important to evaluate the contribution of systemic ropivacaine in the analgesic/anti-hyperalgesic effect of continuous perineural blocks in clinical practice.

\section{METHODS}

\section{Subjects and Study Design}

The experimental protocol was approved by the Kantonale Ethikkommission Zürich, Sonneggstrasse 12, 8091 Zürich, Switzerland, registration nr. StV. 24-2008, Chairperson Prof. R. Maurer on April 21, 2009 and the Swiss regulatory authorities (Swissmedic, 2009 DR 4027). The study was carried out at the Clinical Trials Centre of the University Hospital of Zurich in accordance with the Helsinki Declaration of 1975, as revised in 2000 and 2008, and the guidelines of Good Clinical Practice (Clinicaltrials.gov: \#NCT00900913). Written informed consent was received from all patients before inclusion in this study. Twenty healthy male volunteers were included in this randomized, double-blinded, placebo-controlled, crossover study. Inclusion criteria were male: age $18-65$ years; weight: $50-100 \mathrm{~kg}$; height: $155-195 \mathrm{~cm}$; signed and dated informed consent. Exclusion criteria were contraindications or hypersensitivity to local 
anesthetics, vulnerable subjects (intellectually or mental impaired), drug, alcohol or nicotine abuse, known peripheral neuropathies, diabetes mellitus and/or congestive heart disease. All subjects were familiarized with the tests performed before undergoing two testing sessions at least 1 week apart, receiving ropivacaine and saline as a placebo. Randomization of the sequence of the test drugs was generated with the method of envelopes by an un-blinded research assistance who was not involved in the collecting or the assessment of data.

\section{Electrical Pain Model and 'Pain Tracking'}

An electrical hyperalgesia model was used to induce ongoing pain, hyperalgesia, allodynia, and flare reaction [18]: Two insulated gold wires (AU-3T, $76 \mu \mathrm{m}$, Science Products $\mathrm{GmbH}$, Hofheim, Germany) diameter were inserted $1 \mathrm{~cm}$ intradermally into the left central volar forearm of the subjects. The distance between the un-insulated tips of the two gold wires was $5 \mathrm{~mm}$. Electrical stimuli (rectangular; $1 \mathrm{~ms}$ ) were applied via a constant current stimulator (DS5, Digitimer Ltd., Hertfordshire, UK) at $1 \mathrm{~Hz}$. The study subjects were asked to rate the pain intensity in their arm on a box with a sliding button on a numeric rating scale (NRS; 0: no pain; 10: maximal pain imaginable). The box was connected with a software (QtracW@), Institute of Neurology, London, UK) which 'tracked' the pain intensity and adjusted the stimulus strength according to the rating (Fig. 1). In the current study, we designed the stimulation protocol in a way to inflict a pain intensity of NRS 5. For example, if the subject indicated on the box a pain intensity value less than 5 , the stimulation strength automatically increased in one-percent steps. If the pain intensity was rated more than 5 , stimulation

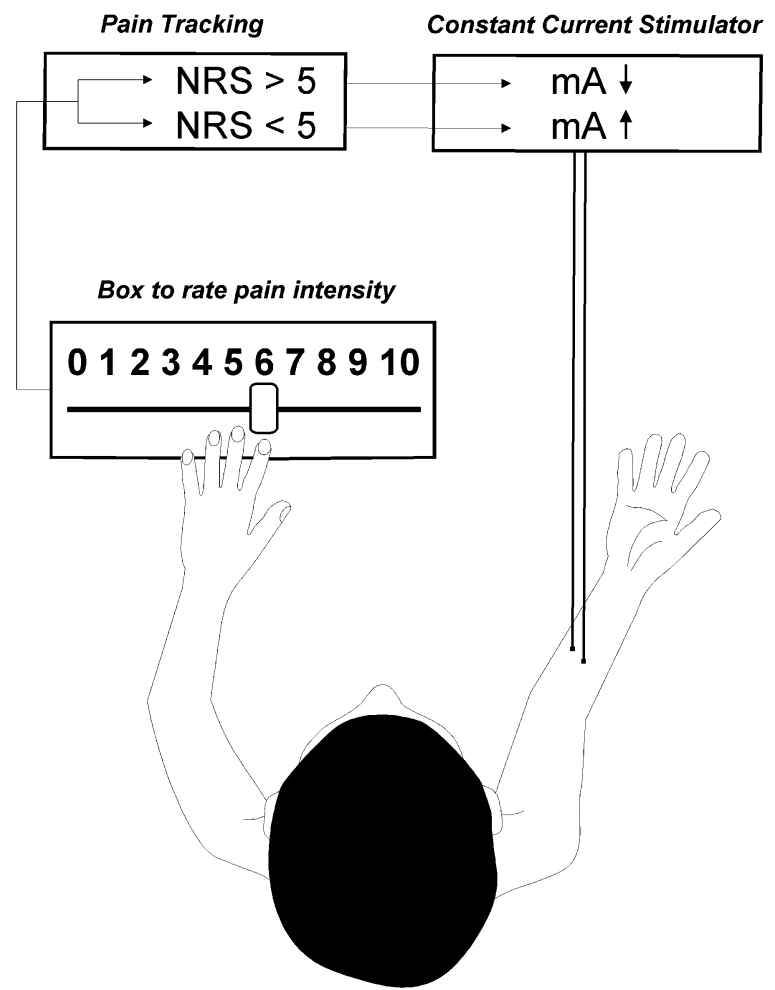

Fig. 1 Pain tracker. The experimental set-up used in this study allowed a constant pain experience by adapting the current strength to the actual rating (pain tracking). Electrical pain was generated by a constant current stimulator on the volar side of one forearm of the study subject. On the other hand, the subjects were rating continuously the intensity of the inflicted pain on a scaled box ( 0 no pain, 10 maximal pain imaginable) with a sliding button. The box was connected to computer software (QTRAC@) which adjusted the stimulator output according to the actual rating of the study subject. If the pain rating was below 5 , stimulus strength was slowly increased. If the pain rating was more than 5 , the stimulus strength slowly decreased. NRS numeric rating scale

strength automatically decreased in onepercent steps. This stimulation approach forced the subjects to rate the pain continuously by means of the box, because the stimulation current was either increasing or decreasing.

The rationale of this setup was based on theoretical and practical advantages. First, we wanted to 'optimize' the development of peripheral and central symptoms by 


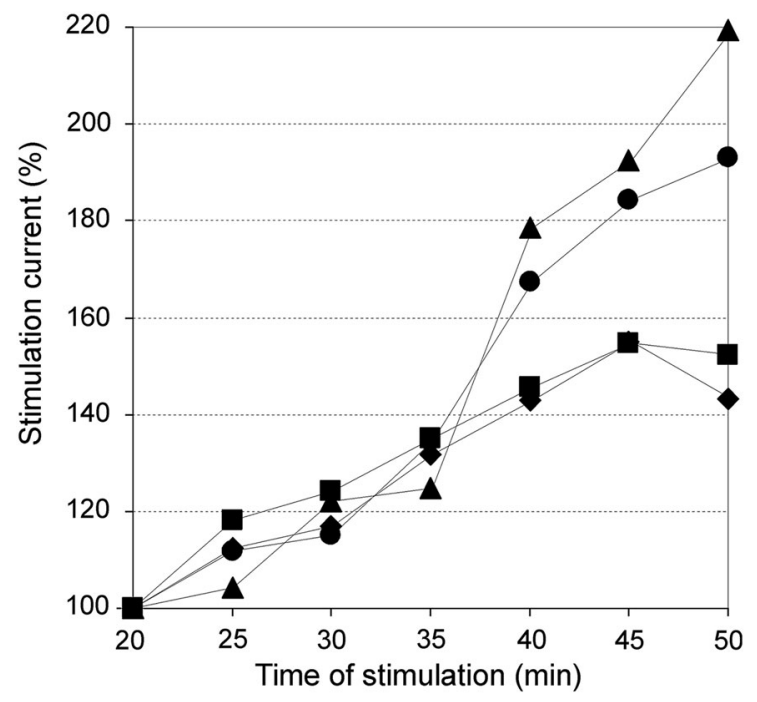

Fig. 2 Current increase over time after $20 \mathrm{~min}$ of stimulation (pilot study, $n=4$ ). In a preliminary set of experiments in four subjects, current increase over time at a numeric rating scale of 5 was recorded. Between 20 and $50 \mathrm{~min}$ of stimulation, all of the subjects increased the stimulation current to at least $140 \%$. Each line represents the normalized current increase in an individual subject

maintaining a constant intensity of the pain stimulus over time and independent of endogenous suppressing mechanisms which were described with this model [19]. Second, rating a constant pain 'experience' where a study subject only has to decide if the pain intensity is stronger or less strong than a defined value is an easier task than using a full numerical rating scale. As a consequence of this 'pain tracking', the stimulation current was expected to increase over time. Preliminary experiments in four pilot subjects showed that after 20 min of stimulation in all of the subjects, the stimulation current increased more than $40 \%$ within the subsequent $30 \mathrm{~min}$ (Fig. 2). Based on this information, we designed the study and defined two reading points (Fig. 3): electrical stimulation was started and continued for $20 \mathrm{~min}$ until $t 0$; the current intensity to maintain an NRS 5 at $t 0$ was defined as $100 \%$

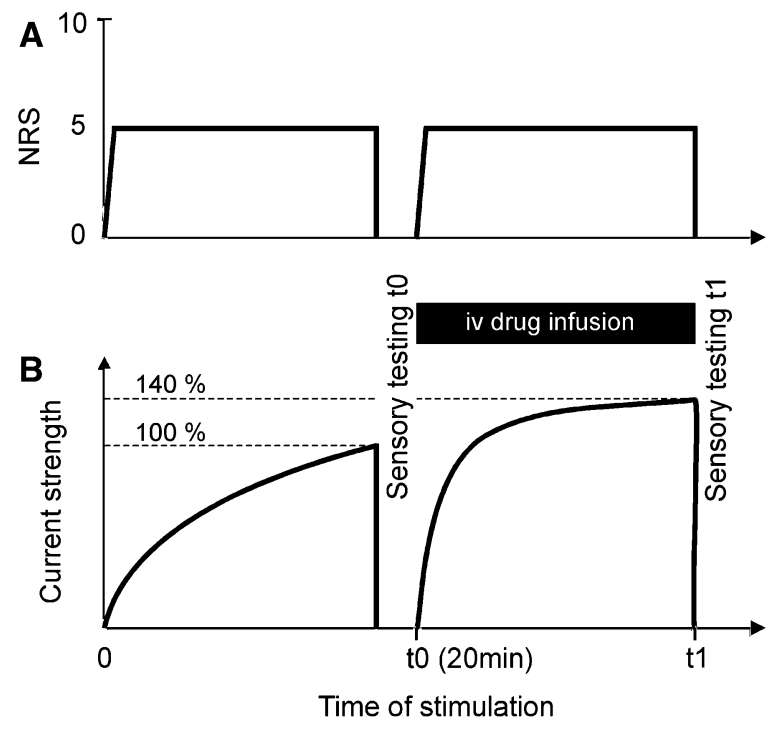

Fig. 3 Time course of experiment. After initial adaptation to the painful stimuli, the subjects were asked to rate their pain intensity on the pain tracking box (see Fig. 1). The pain tracker forced the subject to maintain a pain intensity of 5 (a). To maintain a constant pain rating level, the current strength has to increase over time due to endogenous pain suppressing mechanisms (b). Before starting the drug infusion at $t 0$, a first sensory testing was performed. Stimulation was restarted and the subjects continued rating the pain. The drug was infused at a constant rate until the current strength of the stimulation reached $140 \%(t 1)$ of the current strength defined as $100 \%$ at $t 0$. Subsequently, the second sensory testing was performed. iv intravenous $N R S$ numeric rating scale

and baseline sensory testing was performed (see below). At $t 0$, an infusion of the drug was started (under on-going stimulation) at a constant rate until $t 1$, defined as the time after to when the current intensity reached $140 \%$ (of the intensity at $t 0$ ). One of the endpoints of the study was to measure a possible analgesic effect of systemic ropivacaine on the acute local pain sensation induced by the electrical stimulation. If ropivacaine produced any analgesic effect-so the hypothesis-the stimulation intensity threshold of $140 \%$ would be reached earlier than a placebo infusion. 


\section{Treatments and Dose Rationale}

Administration of systemic ropivacaine is an off-label use of the drug and associated with risks of toxicity [20]. By defining the dosages, particular care was taken to avoid potential dangerous side effects. The calculation was based on previously published data [21].

The treatment infusions were prepared by an independent non-blinded investigator according to the body weight of the study subjects: Ropivacaine $1 \mathrm{mg} / \mathrm{kg}$ (Naropin $\odot$ AstraZeneca AG, Zug, Switzerland), drawn up with $\mathrm{NaCl} 0.9 \%$ to a total volume of $30 \mathrm{ml}$ volume per syringe. Using this approach, the maximum dose infused in a study subject could not reach any dangerous plasma levels if the target threshold of $140 \%$ (see above) was not reached within $30 \mathrm{~min}$. The placebo infusion consisted of $30 \mathrm{ml}$ of $\mathrm{NaCl} 0.9 \% .20 \mathrm{~min}$ after the start of the electrical stimulation $(t 0)$, the subjects received the treatment intravenously delivered with an infusion pump (Alaris ${ }^{\circledR} \mathrm{GH}$ Syringe Pump, Cardinal Health, Rolle, Switzerland) at a rate of $60 \mathrm{ml} / \mathrm{h}$. The infusion of the drug was stopped as soon as the target current of $140 \%$ was reached ( $t 1)$. During the infusion of the drug, the study subjects were regularly checked for side effects, such as pruritus, perioral numbness or tingling, hypoacusis or hyperacusis, dizziness, nausea, sedation, or dissociative effects. Pulse oximetry (oxygen saturation), electrocardiogram, and non-invasive arterial pressure were monitored continuously.

\section{Sensory Testing}

At the reading points $t 0$ and $t 1$, the area of punctate hyperalgesia was measured with a $256 \mathrm{mN}$ von Frey filament (Fruhstorfer, Erlangen, Germany), and the area of dynamic allodynia was determined with a standardized brush (Somedic, Hörby, Sweden) exerting a force of approximately $200-400 \mathrm{mN}$ gently stroked on the skin. The borders of the hyperalgesic areas were delineated by stimulating along four linear paths parallel and vertical to the axis of the forearm from distant starting points toward the stimulation site, until the study subject reported increased pain sensations evoked by the von Frey filament (punctate hyperalgesia) or unpleasant sensations or pain by stroking the skin with brush (allodynia) [18]. These sites were marked on the skin and traced on sheet at the end of the experiment. For further analysis, both diameters were used to calculate the areas of hyperalgesia.

\section{Flare Reaction}

Superficial blood flow of the stimulated arm was measured repetitively by laser Doppler imaging (moorFLIPI, Moor Instruments Ltd., Devon, United Kingdom). For this purpose, an area of $16 \mathrm{~cm}$ by $8 \mathrm{~cm}$ around the injection sites was scanned with a resolution of 49,000 pixels per $\mathrm{cm}^{2}$, with each pixel representing a separate Doppler flux measurement. Data were stored on hard disk and processed offline with dedicated software (MoorLDI Version 5.0, Moor Instruments Ltd.). The flare area was calculated from all pixels around the stimulation site in which flux values exceeded the $95 \%$ percentile of the baseline distribution [22]. Laser Doppler images were recorded before starting the stimulation (baseline image), at the beginning of the infusion ( $t 0)$ and at the end of the infusion ( $t 1)$.

\section{Statistical Analysis}

The hypothesis of this study was that low-dose ropivacaine may affect clinical signs like hyperalgesia, allodynia and flare reaction 
(primary endpoints). Secondary endpoints were time to reach the threshold current and incidence of side effects. To our knowledge, no previous study has investigated these endpoints. For our sample size determination, we, therefore, used an approximation based on data previously published on lidocaine [7]. We calculated a SD of $6.9 \mathrm{~cm}^{2}$ hyperalgesia after 18-min subcutaneous electrical stimulation in a treated and non-treated group. We supposed a mean difference of hyperalgesia of $5.5 \mathrm{~cm}^{2}$ and calculated a power of $90 \%$ for a sample size of

\section{The Consort Flowchart}

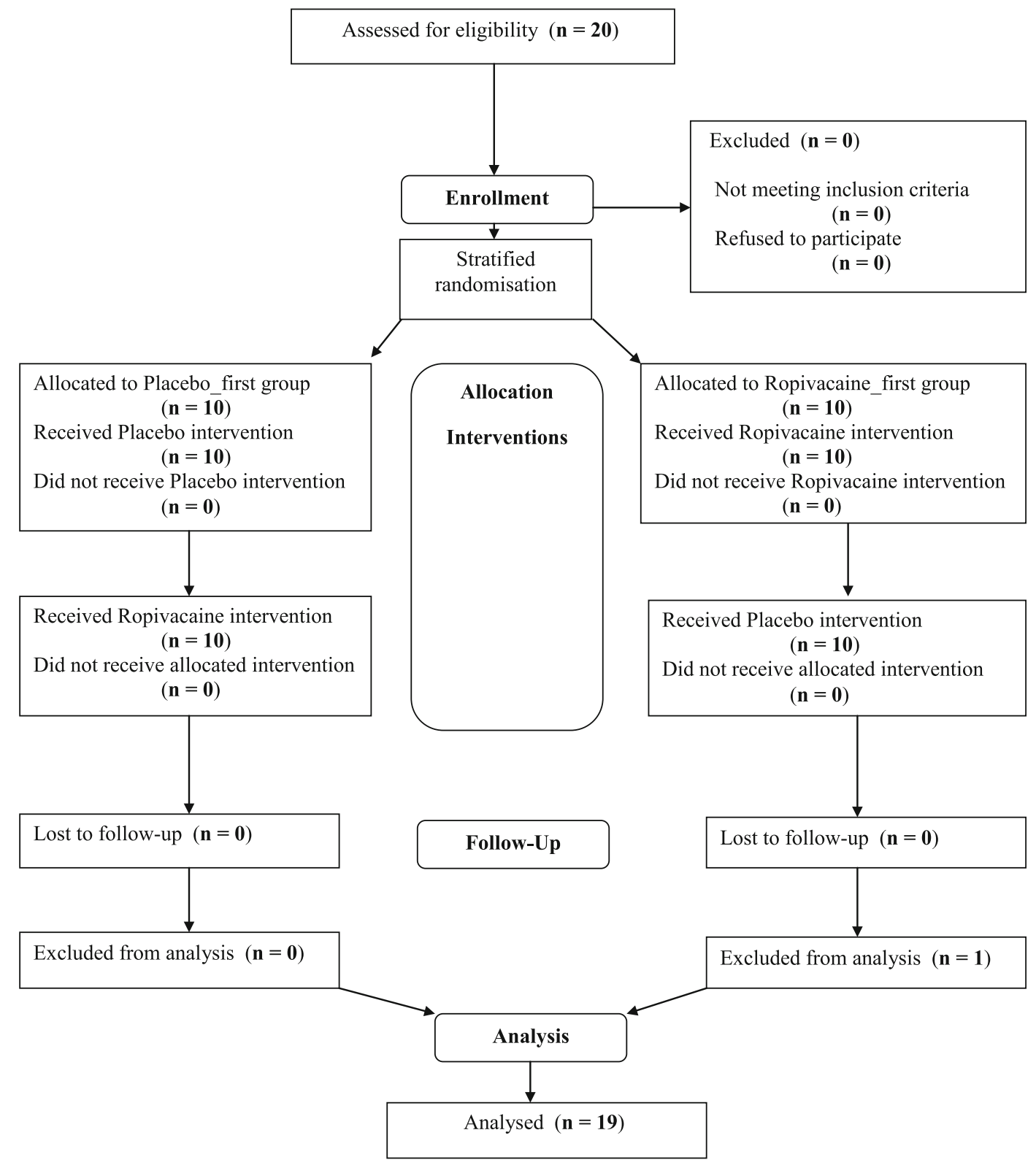

Fig. 4 Flow diagram of the progress through the phases of trial (enrollment, intervention allocation, follow-up, and data analysis) 
19 subjects with a paired $t$ test $(P<0.05)$. All results were expressed as mean $\pm S D$, except for in the figures in which data are presented as mean \pm SE. IBM SPSS Statistics ${ }^{\mathrm{TM}}$ 19.0.1 (SPSS Inc., Chicago, IL, USA) was used for statistical analysis. Stimulation duration to reach $t 1$ was tested with paired $t$ test assuming normal distribution. Data of hyperalgesia, allodynia and flare reaction at $t 0$ and $t 1$ were normalized and then tested with paired $t$ test assuming normal distribution. Side effects were analyzed with McNemar test.

\section{RESULTS}

All 20 subjects included in the investigation finished the study (Fig. 4). One subject was not able to clearly indicate hyperalgesia or allodynia in any of the sessions and the data were not included in the analysis. An overview of the demographic of the study population is given in Table 1. Maintaining a pain intensity of 5 during the stimulation time on the NRS box was considered to be an easy task by all the subjects and was well tolerated.

Table 1 Demographic data

\begin{tabular}{|c|c|c|c|c|c|}
\hline $\begin{array}{l}\text { Study subject } \\
\text { (number) }\end{array}$ & $\begin{array}{l}\text { Age } \\
\text { (years) }\end{array}$ & $\begin{array}{l}\text { Height } \\
\text { (cm) }\end{array}$ & $\begin{array}{l}\text { Weight } \\
(\mathbf{k g})\end{array}$ & $\begin{array}{l}\text { Total ropivacaine } \\
\text { infused (mg) }\end{array}$ & $\begin{array}{l}\text { Ropivacaine } \\
\text { infused }(\mathrm{mg} / \mathrm{kg})\end{array}$ \\
\hline 1 & 39 & 171 & 75 & 55.0 & 0.7 \\
\hline 2 & 26 & 179 & 64 & 42.7 & 0.7 \\
\hline $3^{a}$ & 28 & 170 & 63 & 16.8 & 0.3 \\
\hline 4 & 37 & 179 & 75 & 35.0 & 0.5 \\
\hline 5 & 27 & 189 & 84 & 22.4 & 0.3 \\
\hline 6 & 29 & 180 & 76 & 17.7 & 0.2 \\
\hline 7 & 28 & 186 & 70 & 9.3 & 0.1 \\
\hline 8 & 26 & 179 & 75 & 60.0 & 0.8 \\
\hline 9 & 35 & 183 & 67 & 58.1 & 0.9 \\
\hline 10 & 29 & 172 & 63 & 31.5 & 0.5 \\
\hline 11 & 25 & 174 & 67 & 38.0 & 0.6 \\
\hline 12 & 33 & 171 & 68 & 29.5 & 0.4 \\
\hline 13 & 25 & 190 & 90 & 57.0 & 0.6 \\
\hline 14 & 33 & 178 & 78 & 62.4 & 0.8 \\
\hline 15 & 26 & 172 & 67 & 17.9 & 0.3 \\
\hline 16 & 27 & 175 & 75 & 17.5 & 0.2 \\
\hline 17 & 21 & 184 & 85 & 65.2 & 0.8 \\
\hline 18 & 26 & 177 & 79 & 71.1 & 0.9 \\
\hline 19 & 26 & 186 & 99 & 46.2 & 0.5 \\
\hline 20 & 25 & 183 & 86 & 63.1 & 0.7 \\
\hline Mean \pm SD & $30 \pm 5$ & $179 \pm 6$ & $75.3 \pm 10.0$ & $40.8 \pm 19.7$ & $0.5 \pm 0.2$ \\
\hline
\end{tabular}

a Subject number 3 was excluded from the analysis 


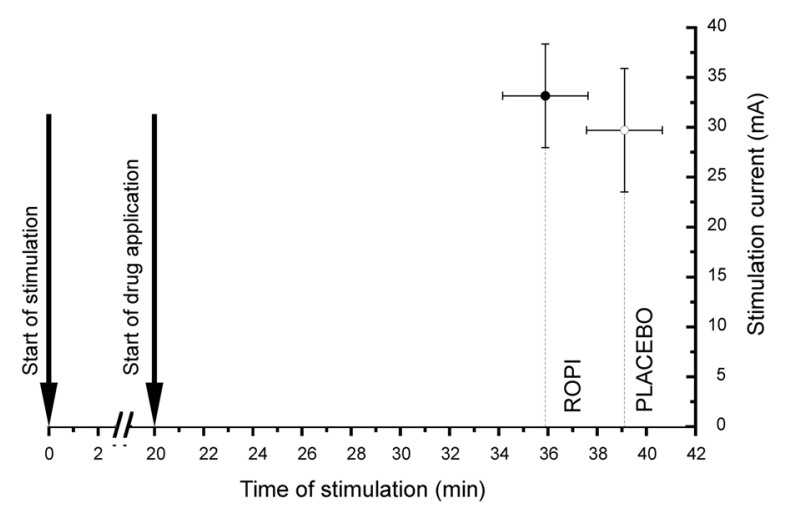

Fig. 5 Time $(t 1)$ to reach threshold current: Effect on acute pain sensation $20 \mathrm{~min}$ after the beginning of the stimulation $(t 0)$, the drugs were infused intravenously. $t 1$ was the time to reach the defined current threshold of $140 \%$ of the current strength at t0. Error bars are SE. During the infusion of ropivacaine, the threshold was reached earlier than during the infusion with placebo; however, this difference was not significant. ROPI ropivacaine

\section{Current Increase Between to and $t 1$}

In all the experiments, the targeted threshold of $140 \%$ of the stimulus strength at $t 0$ was reached within the infusion time of $30 \mathrm{~min}$ (Fig. 5). The current strength at $t 0$ was not different between the two groups. There was no time difference in increase to the target threshold between ropivacaine $(15.9 \pm 7.0 \mathrm{~min})$ and placebo $(19.1 \pm 5.8 \mathrm{~min} ; P=0.10)$.

\section{Hyperalgesia, Allodynia, and Flare Reaction}

The areas of hyperalgesia, allodynia, and flare reaction were not different at $t 0$ in the sessions with placebo or ropivacaine. During the infusions of the drugs, the area of hyperalgesia did increase with placebo $(303 \pm 380 \%, P<0.05)$ and ropivacaine $(186 \pm 137 \%, P<0.05)$ (Fig. 6$)$. The area of allodynia increased significantly during the placebo $(253 \pm 299 \%, \quad P<0.05)$ infusion. We did not find any difference in increase in allodynia during the ropivacaine infusion $(137 \pm 132 \%, P=0.22)$ between $t 0$ and $t 1$. Similar results were found regarding the flare response. The area and intensity of flare increased significantly during the placebo infusion (area: $112 \pm 24 \%, P<0.05$; intensity: $110 \pm 17 \%, P<0.05$ ) but not during the infusion with ropivacaine (area: $107 \pm 1 \%, P=0.31$; intensity: $102 \pm 21 \%, P=0.19)$.

\section{Side Effects}

In nine subjects, we observed side effects (dizziness and/or tingling around the mouth). This was significantly higher than during the placebo $(n=0, P<0.01)$ infusion.

\section{DISCUSSION}

In this investigation, systemic ropivacaine diminished the development of brush-evoked allodynia and flare response but not punctate hyperalgesia or acute pain sensation. These results suggest that ropivacaine had an impact on both, peripheral and central nervous mechanisms.

Hyperalgesia to mechanical stimuli may develop at the site of injury (zone of primary hyperalgesia) and in the uninjured area surrounding an injury (zone of secondary hyperalgesia) [23]. Primary hyperalgesia involves a sensitization of primary sensory afferents in the periphery which supposedly causes local release of inflammatory mediators [24]. In contrast, 'secondary hyperalgesia' is characterized by increased pain intensity from mechanical stimuli only [25] and is considered to be caused by central mechanisms $[26,27]$. As a result, normally painful punctate stimuli become even more painful ('hyperalgesia') and input from normally non-painful stimuli is perceived as painful ('allodynia'). The latter is 

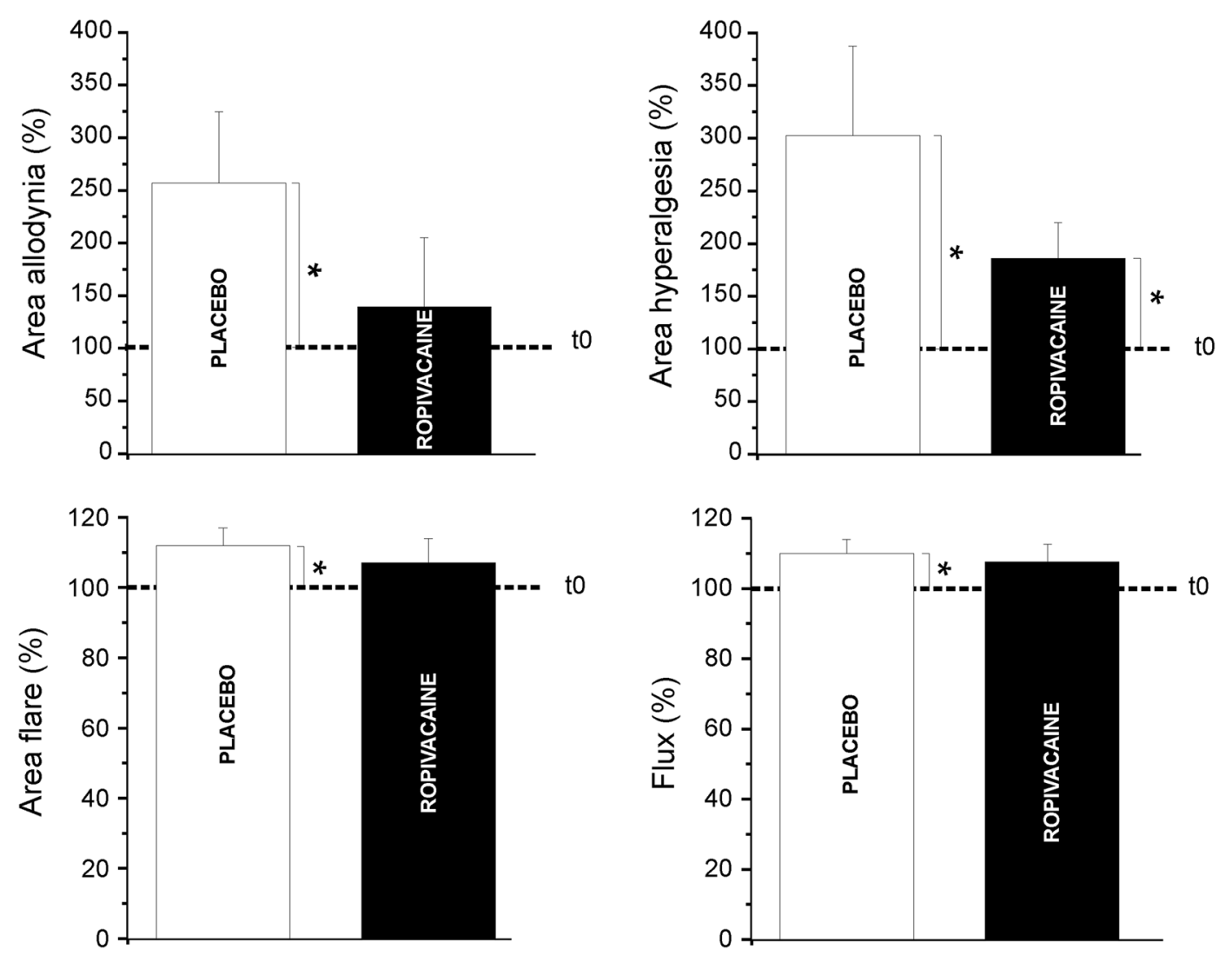

Fig. 6 Areas of hyperalgesia, allodynia, flare reaction, and flare intensity after the drug infusion at $t 1$. The area of allodynia increased significantly with placebo but not with ropivacaine compared to the baseline at t0 (dotted line).

Mechanical hyperalgesia increased significantly with both, ropivacaine and placebo. Area and intensity of flare increased significantly with placebo but not with ropivacaine. Error bars are SE; ${ }^{*} P<0.05$

mediated by low-threshold fibers which are signaling the sensation of touch in normal physiological conditions [28]. The mechanisms are not fully understood but second-order neurons in the central nervous system on the level of the spinal cord play a major role in this process [29].

\section{Peripheral versus Central Effects of Systemic Ropivacaine}

On the level of the nerve endings and the receptive field in the skin, local anesthetics interfere with the activation of nociceptors by blocking voltage-gated sodium channels. This mechanism is mainly responsible for the

reduction in acute pain sensation; hence it represents the typical analgesic effect of local anesthetics in the peripheral nervous system [4]. It is dependent on the concentration of the local anesthetic. In the current study, the concentration reached systemically was probably too low to show any analgesic effect on the painful stimulation. This is in line with previous investigations of low-dose local anesthetics where in healthy volunteers also acute nociception remained intact in the presence of a limited and selective antihyperalgesic effect of lidocaine [17, 30].

However, the flare reaction, a typical peripheral symptom of the electrical stimulation [26], was significantly modulated 
by ropivacaine. Under physiological conditions, activation of nociceptors leads to an erythema in the surrounding of the injury, also called 'flare'. This reaction is caused by mechanoinsensitive nociceptors (also called "silent nociceptors") which trigger the release of vasoactive neuropeptides (bradykinin, calcitonin gene-related peptide, substance P) at the nerve endings ("neurogenic inflammation") when activated. The most likely explanation for a diminished flare response in the presence of local anesthetics is an attenuation of a vascular response to bradykinin and substance P not only when applied locally, but also when applied systemically at low-dose concentrations [14]. Also, local anesthetics tend to cause vasoconstriction which has to be taken into consideration when interpreting flare response [31]. It is postulated that the degree of vasoconstriction depends on the stereoselectivity of the local anesthetic and, therefore, $S$-enantiomers like ropivacaine could have a more pronounced effect than the other local anesthetics [32]. The current study revealed that the flare response was diminished by ropivacaine in terms of area and intensity and, therefore, the results go in line with previous investigations with different systemic sodium channel blockers [7, 14, 17, 33].

Another possible peripheral effect we observed was the diminished development of allodynia. There is abundant evidence that large myelinated fibers are involved in mediating pain in the area of secondary hyperalgesia [34] and allodynia [35]. Low-dose local anesthetics can prevent flow of information (number of action potentials) toward the central nervous system, especially in neuropathic nerves [36]. As a consequence, less neurotransmitter will be released at the central endings in the spinal cord, and nociceptive activity in the dorsal horn will decrease [37]. This diminished activity in the dorsal horn, in turn, will lead to a decrease of 'secondary hyperalgesia' or allodynia on the peripheral end of the nociceptors in the surrounding tissue of the receptive field [6-8].

There are still no clear neurophysiological investigations as to what the exact underlying mechanisms of brush-evoked allodynia are, but an involvement of the central nervous system is undisputed [29]. Therefore, the results of this study would imply that ropivacaine modulated pain pathways also on a central level. Regarding the impact on mechanical hyperalgesia, Hahnenkamp et al. [38] found that $S$ ropivacaine had a larger effect on the inhibition of $\mathrm{N}$-methyl-D-aspartate receptor (also known as the NMDA receptor compared to other local anesthetics. This inhibition would imply an even stronger effect on secondary hyperalgesia, hence on central mechanisms) [38]. The results of this study, however, showed that ropivacaine did not impair the development of punctate hyperalgesia. Since our sensory testing did not differentiate between primary (peripheral mechanisms) and secondary hyperalgesia (central mechanisms), the diminished sensitization processes in the periphery-indirectly illustrated by the impaired flare response-would account solely for an incomplete explanation of the lacking efficacy. Our findings, however, are comparable to a recent publication by Charlet et al. [39]. They found that systemic ropivacaine did not prevent mechanical hyperalgesia compared to locally applied ropivacaine without giving a satisfying explanation of the underlying mechanisms [39].

There is no clear hypothesis as to why ropivacaine would act differently to other sodium channel blockers in terms of central mechanisms. In this study, despite observing a reduced development of punctate hyperalgesia compared to placebo, we assume that the 
concentrations reached intrathecally were not high enough and/or the variance of the electrical model to induce hyperalgesia was too large to detect statistical significant effects.

\section{CONCLUSION}

The results of this study imply that systemic ropivacaine may prevent pain sensitization processes. Clinically, these effects could be relevant since continuous application of ropivacaine (e.g., continuous nerve block after surgery) leads to substantially elevated systemic plasma concentration. The incidence of side effects was nearly 50\% during the infusion with ropivacaine at even low dosages. Therefore, the therapeutic benefit to justify an intravenous use of the drug to treat chronic pain states-as it was proposed by other groups [16]—seems to be very limited. Furthermore, the extrapolation of results in this study with healthy volunteers on neuropathic pain states in patients needs to be verified in a clinical setting. Therefore, more studies with different models of hyperalgesia (e.g., UV model or capsaicin model) and in patients with neuropathic pain are needed.

\section{ACKNOWLEDGMENTS}

This study was supported by the Institute of Anaesthesiology, University Hospital Zurich, Switzerland and the Clinical Trials Centre, University of Zurich, Switzerland. Funding was received from the Swiss Foundation for Anaesthesia Research (SFAR), by the Swiss National Science Foundation (Grant Nr. SPUM 33CM30_124117) and the Institute of Anaesthesiology, University Hospital Zurich, Switzerland. Neither of these sponsors played any role in study design; collection, analysis and interpretation of data; writing of the manuscript; and the decision for submission. No funding was received for processing charges of this article. No medical writing assistance was received for this article. Dr K. Maurer is the guarantor for this article, and takes responsibility for the integrity of the work as a whole.

Conflict of interest. In the past, Patrick Willimann has received travel support for consulting or lecturing from the following companies: Pfizer AG, Zurich, Switzerland; Mundipharma Medical Company, Basel, Switzerland; Medtronic, Bern, Switzerland; Grünenthal Pharma Schweiz, Mitlödi; Switzerland; St. Jude Medical AG, Zurich, Switzerland.

Donat Spahn's academic department is receiving grant support from the Swiss National Science Foundation, Berne, Switzerland (grant numbers: 33CM30_124117 and 406440131268), the Swiss Society of Anaesthesiology and Reanimation (SGAR), Berne, Switzerland (no grant numbers are attributed), the Swiss Foundation for Anaesthesia Research, Zurich, Switzerland (no grant numbers are attributed), Bundesprogramm Chancengleichheit, Berne, Switzerland (no grant numbers are attributed), CSL Behring, Berne, Switzerland (no grant numbers are attributed), Vifor SA, Villarssur-Glâne, Switzerland (no grant numbers are attributed). Dr. Spahn was the chairman of the $\mathrm{ABC}$ Faculty and is a member of the $\mathrm{ABC}$ Trauma Faculty which both are managed by Thomson Physicians World GmbH, Mannheim, Germany and sponsored by an unrestricted educational grant from Novo Nordisk A/S, Bagsvärd, Denmark and CSL Behring GmbH, Hattersheim am Main, Germany. In the past 5 years, Dr. Spahn has received honoraria or travel support for consulting or lecturing from the following companies: Abbott AG, Baar, 
Switzerland, AstraZeneca AG, Zug, Switzerland, Bayer (Schweiz) AG, Zürich, Switzerland, Baxter S.p.A., Roma, Italy, B. Braun Melsungen AG, Melsungen, Germany, Boehringer Ingelheim (Schweiz) GmbH, Basel, Switzerland, BristolMyers-Squibb, Rueil-Malmaison Cedex, France, CSL Behring GmbH, Hattersheim am Main, Germany and Bern, Switzerland, Curacyte AG, Munich, Germany, Ethicon Biosurgery, Sommerville, New Jersey, USA, Fresenius SE, Bad Homburg v.d.H., Germany, Galenica AG, Bern, Switzerland (including Vifor SA, Villars-sur-Glâne, Switzerland), GlaxoSmithKline GmbH \& Co. KG, Hamburg, Germany, JanssenCilag AG, Baar, Switzerland, Janssen-Cilag EMEA, Beerse, Belgium, Merck Sharp \& Dohme-Chibret AG, Opfikon-Glattbrugg, Switzerland, Novo Nordisk A/S, Bagsvärd, Denmark, Octapharma AG, Lachen, Switzerland, Organon AG, Pfäffikon/SZ, Switzerland, Oxygen Biotherapeutics, Costa Mesa, CA, Pentapharm $\mathrm{GmbH}$ (now tem Innovations $\mathrm{GmbH}$ ), Munich, Germany, Roche Pharma (Schweiz) AG, Reinach, Switzerland and Schering-Plough International, Inc., Kenilworth, New Jersey, USA.

In the past, Konrad Maurer has received travel support for consulting or lecturing from the following companies: Pfizer AG, Zurich, Switzerland; Bristol-Myers Squibb SA, Baar, Switzerland; Mundipharma Medical Company, Basel, Switzerland; Janssen-Cilag AG, Baar, Switzerland; UCB, Bulle, Switzerland; Medtronic, Bern, Switzerland; B. Braun Medical AG, Sempach, Switzerland; Grünenthal Pharma Schweiz, Mitlödi; Switzerland; St. Jude Medical AG, Zurich, Switzerland.

Yéri Haller and Andreas Gantenbein declare no conflict of interest.

Ethical standard. The experimental protocol was approved by the Kantonale Ethikkommission
Zürich, Sonneggstrasse 12, 8091 Zürich, Switzerland, registration nr. StV. 24-2008, Chairperson Prof. R. Maurer on April 21, 2009 and the Swiss regulatory authorities (Swissmedic, 2009 DR 4027). The study was carried out at the Clinical Trials Centre of the University Hospital of Zurich in accordance with the Helsinki Declaration of 1975, as revised in 2000 and 2008, and the guidelines of Good Clinical Practice (Clinicaltrials.gov: \#NCT00900913). Written informed consent was received from all patients before inclusion in this study.

Open Access. This article is distributed under the terms of the Creative Commons Attribution Noncommercial License which permits any noncommercial use, distribution, and reproduction in any medium, provided the original author(s) and the source are credited.

\section{REFERENCES}

1. Grossi P, Urmey WF. Peripheral nerve blocks for anaesthesia and postoperative analgesia. Curr Opin Anaesthesiol. 2003;16:493-501.

2. Schug SA, Fry RA. Continuous regional analgesia in comparison with intravenous opioid administration for routine postoperative pain control. Anaesthesia. 1994;49(6):528-32.

3. Ekatodramis G, Borgeat A, Huledal G, Jeppsson L, Westman L, Sjovall J. Continuous interscalene analgesia with ropivacaine $2 \mathrm{mg} / \mathrm{ml}$ after major shoulder surgery. Anesthesiology. 2003;98:43-50.

4. Strichartz G. Molecular mechanisms of nerve block by local anesthetics. Anesthesiology. 1976;45: 421-41.

5. Koppert W, Brueckl V, Weidner C, Schmelz M. Mechanically induced axon reflex and hyperalgesia in human UV-B burn are reduced by systemic lidocaine. Eur J Pain. 2004;8:237-44.

6. Abram SE, Yaksh TL. Systemic lidocaine blocks nerve injury-induced hyperalgesia and nociceptordriven spinal sensitization in the rat. Anesthesiology. 1994;80:383-91. 
7. Koppert W, Ostermeier N, Sittl R, Weidner C, Schmelz M. Low-dose lidocaine reduces secondary hyperalgesia by a central mode of action. Pain. 2000;85:217-24.

8. Klede M, Handwerker HO, Schmelz M. Central origin of secondary mechanical hyperalgesia. J Neurophysiol. 2003;90:353-9.

9. Kuo CP, Jao SW, Chen KM, et al. Comparison of the effects of thoracic epidural analgesia and i.v. infusion with lidocaine on cytokine response, postoperative pain and bowel function in patients undergoing colonic surgery. $\mathrm{Br} \mathrm{J}$ Anaesth. 2006;97:640-6.

10. Herroeder S, Pecher S, Schonherr ME, et al. Systemic lidocaine shortens length of hospital stay after colorectal surgery: a double-blinded, randomized, placebo-controlled trial. Ann Surg. 2007;246: 192-200.

11. Radwan IA, Saito S, Goto F. The neurotoxicity of local anesthetics on growing neurons: a comparative study of lidocaine, bupivacaine, mepivacaine, and ropivacaine. Anesth Analg. 2002;94:319-24.

12. Casati A, Putzu M. Bupivacaine, levobupivacaine and ropivacaine: are they clinically different? Best Pract Res Clin Anaesthesiol. 2005;19:247-68.

13. Graf BM, Abraham I, Eberbach N, Kunst G, Stowe DF, Martin E. Differences in cardiotoxicity of bupivacaine and ropivacaine are the result of physicochemical and stereoselective properties. Anesthesiology. 2002;96:1427-34.

14. Dias MP, Newton DJ, McLeod GA, Khan F, Belch JJ. The inhibitory effects of local anaesthetics on the vascular flare responses to bradykinin and substance $P$ in human skin. Anaesthesia. 2008;63:151-5.

15. Kfoury T, Mazoit JX, Schumacher M, Benhamou D, Beloeil H. A comparison of different dosages of a continuous preperitoneal infusion and systemic administration of ropivacaine after laparotomy in rats. Anesth Analg. 2011;113:617-25.

16. Sato C, Sakai A, Ikeda Y, Suzuki H, Sakamoto A. The prolonged analgesic effect of epidural ropivacaine in a rat model of neuropathic pain. Anesth Analg. 2008;106:313-20.

17. Koppert W, Zeck S, Sittl R, Likar R, Knoll R, Schmelz M. Low-dose lidocaine suppresses experimentally induced hyperalgesia in humans. Anesthesiology. 1998;89:1345-53.

18. Koppert W, Dern SK, Sittl R, Albrecht S, Schuttler J, Schmelz M. A new model of electrically evoked pain and hyperalgesia in human skin: the effects of intravenous alfentanil, $\mathrm{S}(+)$-ketamine, and lidocaine. Anesthesiology. 2001;95:395-402.

19. Koppert W, Filitz J, Troster A, et al. Activation of naloxone-sensitive and -insensitive inhibitory systems in a human pain model. J Pain. 2005;6:757-64.

20. Zink W, Graf BM. The toxicity of local anesthetics: the place of ropivacaine and levobupivacaine. Curr Opin Anaesthesiol. 2008;21:645-50.

21. Knudsen K, Beckman Suurkula M, Blomberg S, Sjovall J, Edvardsson N. Central nervous and cardiovascular effects of i.v. infusions of ropivacaine, bupivacaine and placebo in volunteers. Br J Anaesth. 1997;78:507-14.

22. Chizh BA, Dusch M, Puthawala M, et al. The effect of intravenous infusion of adenosine on electrically evoked hyperalgesia in a healthy volunteer model of central sensitization. Anesth Analg. 2004;99:816-22.

23. Raja SN, Campbell JN, Meyer RA. Evidence for different mechanisms of primary and secondary hyperalgesia following heat injury to the glabrous skin. Brain. 1984;107:1179-88.

24. Sauerstein K, Klede M, Hilliges M, Schmelz M. Electrically evoked neuropeptide release and neurogenic inflammation differ between rat and human skin. J Physiol. 2000;15(529 Pt 3):803-10.

25. Ali Z, Meyer RA, Campbell JN. Secondary hyperalgesia to mechanical but not heat stimuli following a capsaicin injection in hairy skin. Pain. 1996;68:401-11.

26. LaMotte RH, Shain CN, Simone DA, Tsai EF. Neurogenic hyperalgesia: psychophysical studies of underlying mechanisms. J Neurophysiol. 1991;66:190-211.

27. Pedersen JL, Crawford ME, Dahl JB, Brennum J, Kehlet $H$. Effect of preemptive nerve block on inflammation and hyperalgesia after human thermal injury. Anesthesiology. 1996;84:1020-6.

28. Cervero F, Laird JM. Mechanisms of touch-evoked pain (allodynia): a new model. Pain. 1996;68(1):13-23.

29. Meyer RA, Ringkamp M, Campbell JN, Raja SN. Peripheral mechanisms of cutaneous nociception. In: McMahon SB, Koltzenburg M, editors. Wall and Melzack's Textbook of Pain. 5th ed. Philadelphia: Elsevier; 2005. p. 3-34.

30. Dirks J, Fabricius P, Petersen KL, Rowbotham MC, Dahl JB. The effect of systemic lidocaine on pain and secondary hyperalgesia associated with the 
heat/capsaicin sensitization model in healthy volunteers. Anesth Analg. 2000;91:967-72.

31. Wallace MS, Laitin S, Licht D, Yaksh TL. Concentration-effect relations for intravenous lidocaine infusions in human volunteers: effects on acute sensory thresholds and capsaicin-evoked hyperpathia. Anesthesiology. 1997;86:1262-72.

32. Iida H, Watanabe $Y$, Dohi S, Ishiyama T. Direct effects of ropivacaine and bupivacaine on spinal pial vessels in canine. Assessment with closed spinal window technique. Anesthesiology. 1997;87:75-81.

33. Kawamata M, Takahashi T, Kozuka Y, et al. Experimental incision-induced pain in human skin: effects of systemic lidocaine on flare formation and hyperalgesia. Pain. 2002;100:77-89.

34. Torebjork HE, Lundberg LE, LaMotte RH. Central changes in processing of mechanoreceptive input in capsaicin-induced secondary hyperalgesia in humans. J Physiol. 1992;448:765-80.

35. Koltzenburg M, Lundberg LE, Torebjork HE. Dynamic and static components of mechanical hyperalgesia in human hairy skin. Pain. 1992;51:207-19.

36. Abdi S, Lee DH, Chung JM. The anti-allodynic effects of amitriptyline, gabapentin, and lidocaine in a rat model of neuropathic pain. Anesth Analg. 1998;87:1360-6.

37. Sotgiu ML, Lacerenza M, Marchettini P. Effect of systemic lidocaine on dorsal horn neuron hyperactivity following chronic peripheral nerve injury in rats. Somatosens Mot Res. 1992;9:227-33.

38. Hahnenkamp K, Durieux ME, Hahnenkamp A, et al. Local anaesthetics inhibit signalling of human NMDA receptors recombinantly expressed in Xenopus laevis oocytes: role of protein kinase $\mathrm{C}$. $\mathrm{Br}$ J Anaesth. 2006;96:77-87.

39. Charlet A, Rodeau JL, Poisbeau P. Radiotelemetric and symptomatic evaluation of pain in the rat after laparotomy: long-term benefits of perioperative ropivacaine care. J Pain. 2011;12:246-56. 